\title{
16.
}

\section{Abrifs einer Theorie der elliptischen Functionen.}

\author{
(Von Herrn Prof. E. Heine zu Bonn.)
}

Im 32ten Bande des gegenwärtigen Journals erwähnt Jacobi, dafs er in seinen Vorlesungen über elliptische Functionen von den $\Theta$ auszugehen pflege, und deutet das Verfahren an, durch welches er die wesentlichsten von den Formeln ableitet, welche in seinem Werke „Fundamenta nova theoriae functionum ellipticarum" zu finden sind. Auch in dem vorliegenden Abrifs ist der Weg verlassen, welcher in den „Fundamentis" eingeschlagen wurde: ich versuche die wesentlichslen Puncte der Theorie möglichst elementar festzustellen, indem ich mich der Methode anschliefse, nach welcher man die Hauptbeziehungen der Kreisfunclionen aus den Reihen zu entwickeln pflegt. Zu letzterem Zwecke stellt man die Reihen auf, welche gleich dem Sinus und Cosinus von $x$ sind, und zeigt durch Quadriren, dafs $\cos ^{2} x+\sin ^{2} x$ gleich 1 ist, d. h. dafs $\cos x$ eine Function von $\sin x$, nemlich $\gamma\left(1-\sin ^{2} x\right)$ ist. Beweiset man ferner durch einfache Differentiation, dafs

$$
\frac{d \sin x}{d x}=\cos x
$$

so ist klar, dafs

$$
x=\int \frac{d(\sin x)}{\sqrt{\left(1-\sin ^{2} x\right)}},
$$

wo das Integral so zu nehmen ist, dafs es für $\sin x=0$ verschwindet, indem die Reihe, welche mit $\sin x$ bezeichnet war, mit $x$ zugleich Null wird. In ähnlicher Weise kann man hier die drei Reihen für $\sin$ am $x, \cos$ am $x, \Delta$ am $x$ aufstellen, und durch Quadriren leicht zeigen, dafs

$$
\begin{aligned}
\cos \text { am } x & =\sqrt{ }\left(1-\sin ^{2} \text { am } x\right), \\
\Delta \text { am } x & =\sqrt{ }\left(1-k^{2} \sin ^{2} \text { am } x\right),
\end{aligned}
$$

wo $k$ eine gewisse Constante (nach $x$ ) bezeichnet. Fügt man hinzu, dafs die zweite Reihe $(\cos a m x)$, mit der dritten $(\Delta \mathrm{am} x)$ multiplicirt, den Differentialquotienten der ersten nach $x$ giebt $\left(\frac{d \sin a m x}{d x}\right)$, dafs ferner $\sin$ am $x$ mit $x$ verschwindet, so erhält man die bekannte Relation zwischen dem elliptischen 
Integral erster Gattung und seiner oberen Grenze, dafs nämlich, wenn

$$
x=\int_{0}^{z} \frac{d z}{\sqrt{\left(1-z^{2}\right) \sqrt{ }\left(1-k^{2} z^{2}\right)}}
$$

gesetzt wird, $z$ von $x$ so abhängt, wie in der ersten Reihe $\sin$ am $x$ von $x$. Diese Entwickelungen findet man im II. Abschnitt. Im III. Abschnitt sind die Formeln für die Addition der elliptischen Functionen durch einfache Multiplication von Reihen abgeleitet; in ähnlicher Weise, wie man die Ausdrücke für die Addition der trigonometrischen Functionen aus den Reihen, durch die sie dargestellt werden, zu beweisen pflegt.

Sind einmal die drei Reihen aufgestell, so scheint mir die im Vorhergehenden angedeutete Art der Untersuchung nicht zu fern zu liegen. Es liegt nahe, jede Reibe mit sich selbst, und darauf mit einer von den beiden andern zu multipliciren; eben so nahe, nachher drei mit einander zu verbinden. Die erste Art von Combinationen verschafft die Entwickelungen des II. Abschnitts, die zweite Art die des dritten. Auf die drei Reihen, so wie auf die übrigen ihnen verwandten führt aber der Gedanke, die Reihen für $\sin x, \cos x$, $\operatorname{arctang} x$, etc. durch Einführung einer neuen Gröfse y, nach dem Princip zu verallgemeinern, welches in meiner Abhandlung in diesem Journal Bd. 34. S. 285 angewandt wurde, um aus der Gau/sischen hypergeometrischen Reihe eine neue zu bilden. Dem aufmerksamen Geometer wird die einfache Form der aus solcher Übertragung entstehenden Ausdrücke ein hinreichender Grund zu ihrer weiteren Untersuchung sein. Nur zur leichteren Darstellung sind sogleich drei zusammengehörige Reihen an die Spitze des II. Abschnitts gestellt: eine Reihe hätte genügt, zu der man die beiden andern ohne Mühe findet.

Anstatt die drei Reihen ferlig aufzustellen, leite ich sie im I. Abschnitt aus den unendlichen Producten durch eine allgemeine Formel ab, deren Beweis dort im Zusammenhange zu finden ist, obgleich er schon in der vorerwähnten Abhandlung gegeben wurde. Die Untersuchungen der beiden letzten Abschnitte sind übrigens im wesentlichen von denen des ersten unabhängig; durch ihn erhalten aber die Formeln die elegante Gestalt, in welcher sie in den „Fundamentis" vorkommen. Die Lehre von der Multiplicalion*) der elliptischen

*) In der oben erwähnten Abhandlung komme ich auf die Multiplication der elliptischen Functionen, indem ich für die Gaufsische Formel

$$
n^{n z} \Pi z \Pi\left(z-\frac{1}{n}\right) \Pi\left(z-\frac{2}{n}\right) \ldots \Pi\left(z-\frac{n-1}{n}\right)=\frac{(2 \pi)^{\frac{1}{2}(n-1)}}{\sqrt{ }(n)} \Pi n z
$$

Crelle's Journal f. d. M. Bd. XXXIX, Hẹt 2 . 
Functionen, wie sie Jacobi in diesem Journal Band 3. Seite 305 angedeutet hat, würde, wenn es die Absicht gewesen wäre, hier eine vollständige Theorie zu geben, am Ende des ersten Abschnitts eingeschaltet worden sein. Auch die Entwickelung der elliptischen Functionen in Kettenbrüche ist hier fortgelassen, da es nicht zweckmäfsig schien, die hierher gehörigen Formeln von den allgemeinen zu trennen, die an einer andern Stelle abgeleitet wurden.

\section{A b s c h n i t t.}

Der Ausdruck

$$
\frac{\left(1-2 q \cos 2 x+q^{2}\right)\left(1-2 q^{3} \cos 2 x+q^{6}\right)\left(1-2 q^{5} \cos 2 x+q^{10}\right) \ldots}{\left(1-2 q^{\alpha+1} \cos 2 x+q^{2 \alpha+2}\right)\left(1-2 q^{\alpha+3} \cos 2 x+q^{2 \alpha+6}\right)\left(1-2 q^{\alpha+5} \cos 2 x+q^{2 \alpha+10}\right) \ldots},
$$

in welchem $q$ und $x$ reelle oder imaginäre Gröfsen bezeichnen, von denen die erstere, wenn sie reell, und ihr Modulus, wenn sie imaginär ist, kleiner als 1 sein mag, läfst sich leicht in eine nach Cosinus der Vielfachen von $x$ fortschreitende Reihe entwickeln. Setzt man dazu $z=e^{2 i x}$, so ist (1.) das Product der beiden Ausdrücke

$$
\frac{(1-q z)\left(1-q^{3} z\right) \ldots}{\left(1-q^{\alpha+1} z\right)\left(1-q^{\alpha+3} z\right) \ldots} \text { und } \frac{\left(1-\frac{q}{z}\right)\left(1-\frac{q^{3}}{z}\right) \ldots}{\left(1-\frac{q^{\alpha+1}}{z}\right)\left(1-\frac{q^{\alpha+3}}{z}\right) \ldots}
$$

von denen sich der erste durch eine nach aufsteigenden, der zweite durch eine nach fallenden Potenzen von $z$ geordnete Reihe darstellen läfst. Es ist nämlich

(2.) $\frac{(1-q z)\left(1-q^{3} z\right) \ldots .}{\left(1-q^{\alpha+1} z\right)\left(1-q^{\alpha+3} z\right) \ldots .}=1+\frac{\left(1-q^{-\alpha}\right)}{\left(1-q^{2}\right)} q^{\alpha+1} z+\frac{\left(1-q^{-\alpha}\right)\left(1-q^{2-\alpha}\right)}{\left(1-q^{2}\right)\left(1-q^{4}\right)} q^{2(\alpha+1)} z^{2}+\ldots$,

so dafs wir als Factor von $2 \cos 2 m x$ in der Entwickelung von (1.), wenn $m$ irgend eine ganze positive Zahl vorstellt, Folgendes erhalten:

$$
\begin{gathered}
\frac{\left(1-q^{-\alpha}\right)\left(1-q^{2-\alpha}\right) \ldots .\left(1-q^{2 m-2-\alpha}\right)}{\left(1-q^{2}\right)\left(1-q^{4}\right) \ldots .\left(1-q^{2 m}\right)} q^{m(\alpha+1)}\left\{1+\frac{\left(1-q^{-\alpha}\right)\left(1-q^{2 m-\alpha}\right)}{\left(1-q^{2}\right)\left(1-q^{2 n+2}\right)} q^{2(\alpha+1)}\right. \\
\left.+\frac{\left(1-q^{-\alpha}\right)\left(1-q^{2-\alpha}\right)\left(1-q^{2 m-\alpha}\right)\left(1-q^{2 m+2-\alpha}\right)}{\left(1-q^{2}\right)\left(1-q^{4}\right)\left(1-q^{2 m+2}\right)\left(1-q^{2 m+4}\right)} q^{4(\alpha+1)}+\ldots . .\right\} .
\end{gathered}
$$

die zwei analogen (90.) und (91.) suche, von denen die erste die eine Transformation, die zweite ihre supplementäre giebt. Die Endresultate (93.) und (94.) sind dort unrichtig angegeben. Man vergleiche das am Ende dieser Abhandlung befindliche Verzeichnifs einiger in jener vorkommenden Errata. 
Die in der Parenthese eingeschlossene Reihe gehört zu der allgemeinen Classe derer, von welchen in einer früheren Abhandlung gezeigt wurde, dafs sie sich durch ein im Allgemeinen unendliches Product summiren lassen. Macht man nämlich $\varphi(a, b, c)=1+\frac{\left(1-q^{2 a}\right)\left(1-q^{2 b}\right)}{\left(1-q^{2}\right)\left(1-q^{2 c}\right)} x+\frac{\left(1-q^{2 a}\right)\left(1-q^{2 a+2}\right)\left(1-q^{2 b}\right)\left(1-q^{2 b+2}\right)}{\left(1-q^{2}\right)\left(1-q^{4}\right)\left(1-q^{2 c}\right)\left(1-q^{2 c+2}\right)} x^{2}+\ldots$. so findet man durch einfache Subtraction, dafs die Differenz

$$
q^{2(a+b-c)} x\left(1-q^{2 c-2 b}\right) \varphi(a+1, b, c+1)-\left(1-q^{2 c}\right) \varphi(a, b, c)
$$

sich als Product von $\left(1-q^{2 c}\right) \varphi(a+1, b, c)$ in $\left(q^{2(a+b-c)} x-1\right)$ darstellen läfst, dafs sie also für $x=q^{2(c-a-b)}$ verschwindet, wenn $q^{c-a-b}$ kleiner als 1 ist. Für diesen Werth von $x$ erhält man demnach

$$
\varphi(a, b, c)=\frac{\left(1-q^{2 c-2 b}\right)}{\left(1-q^{2} c\right)} \varphi(a+1, b, c+1),
$$

und durch wiederholte Anwendung derselben Formel (indem

$$
\varphi(\infty, b, \infty)=1+\frac{\left(1-q^{2 b}\right)}{\left(1-q^{2}\right)} q^{2(c-a-b)}+\frac{\left(1-q^{2 b}\right)\left(1-q^{2 b+2}\right)}{\left(1-q^{2}\right)\left(1-q^{4}\right)} q^{4(c-a-b)}+\ldots
$$

nach (2.) gleich ist $\left.\frac{\left(1-q^{2 c-2 a}\right)\left(1-q^{2 c+2-2 a}\right) \ldots .}{\left(1-q^{2 c-2 a-2 b}\right)\left(1-q^{2 c+2-2 a-2 b}\right) \ldots}\right)$ :

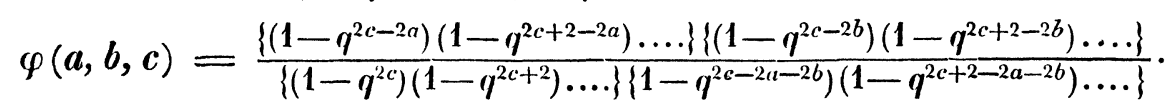

Man hat nur $a=-\frac{1}{2} \alpha, b=m-\frac{1}{2} \alpha, c=m+1$ zu machen, und findet dann für den Factor von $2 \cos 2 m x$ den Werth

$q^{m(\alpha+1)} \cdot \frac{\left(1-q^{-\alpha}\right)\left(1-q^{2-\alpha}\right) \ldots\left(1-q^{2 m-2-\alpha}\right)}{\left(1-q^{2}\right)\left(1-q^{4}\right) \ldots\left(1-q^{2 m}\right)} \cdot \frac{\left(1-q^{\alpha+2}\right)\left(1-q^{\alpha+4}\right) \ldots}{\left(1-q^{2 m+2}\right)\left(1-q^{2 m+4}\right) \ldots} \cdot \frac{\left(1-q^{\alpha+2 m+2}\right)\left(1-q^{\alpha+2 m+4}\right) \ldots .}{\left(1-q^{2 \alpha+2}\right)\left(1-q^{2 \alpha+4}\right) \ldots}$, also schliefslich

$$
\begin{gathered}
\frac{\left\{\left(1-q^{\alpha+2}\right)\left(1-q^{\alpha+4}\right)\left(1-q^{\alpha+6}\right) \ldots\right\}^{2}}{\left.\left\{\left(1-q^{2}\right)\left(1-q^{4}\right) \ldots .\right\}\left\{1-q^{2 \alpha+2}\right)\left(1-q^{2 \alpha+4}\right) \ldots .\right\}}\left\{1+2 \frac{\left(1-q^{-\alpha}\right)}{\left(1-q^{\alpha+2}\right)} q^{\alpha+1} \cos 2 x\right. \\
\left.+2 \frac{\left(1-q^{-\alpha}\right)\left(1-q^{2-\alpha}\right)}{\left(1-q^{\alpha+2}\right)\left(1-q^{\alpha+4}\right)} q^{2(\alpha+1)} \cos 4 x+\ldots .\right\} .
\end{gathered}
$$

Aus dieser allgemeinen Formel lassen sich ohne Mühe die folgenden ableiten:

$$
\begin{gathered}
\text { (4.) } \quad\left\{\left(1-q^{2}\right)\left(1-q^{4}\right) \ldots\right\}\left\{\left(1-2 q \cos 2 x+q^{2}\right)\left(1-2 q^{3} \cos 2 x+q^{6}\right) \ldots\right\} \\
=1-2 q^{1} \cos 2 x+2 q^{4} \cos 4 x-2 q^{9} \cos 6 x+\ldots . \\
\text { (5.) } \quad\left\{\left(1-q^{2}\right)\left(1-q^{4}\right) \ldots\right\} \sin x\left\{\left(1-2 q^{2} \cos 2 x+q^{4}\right)\left(1-2 q^{4} \cos 2 x+q^{8}\right) \ldots\right\} \\
=\sin x-q^{1.2} \sin 3 x+q^{2.3} \sin 5 x-q^{3.4} \sin 7 x+\ldots .
\end{gathered}
$$


(6.) $\quad\left\{\frac{\left(1-q^{2}\right)\left(1-q^{4}\right) \ldots}{(1-q)\left(1-q^{3}\right) \ldots}\right\}^{2} \frac{\left(1-2 q \cos 2 x+q^{2}\right)\left(1-2 q^{3} \cos 2 x+q^{6}\right) \ldots}{\sin x\left(1-2 q^{2} \cos 2 x+q^{4}\right)\left(1-2 q^{4} \cos 2 x+q^{8}\right) \ldots}$

$$
=\frac{1}{\sin x}+\frac{4 q}{1-q} \sin x+\frac{4 q^{3}}{1-q^{3}} \sin 3 x+\ldots
$$

(7.) $\quad \sqrt{ } q\left\{\frac{\left(1-q^{2}\right)\left(1-q^{4}\right) \ldots}{(1-q)\left(1-q^{3}\right) \ldots .}\right\}^{2} \frac{\sin x\left(1-2 q^{2} \cos 2 x+q^{4}\right)\left(1-2 q^{4} \cos 2 x+q^{8}\right) \ldots}{\left(1-2 q \cos 2 x+q^{2}\right)\left(1-2 q^{3} \cos 2 x+q^{6}\right) \ldots}$

$$
=\frac{\sqrt{ } q}{1-q} \sin x+\frac{\sqrt{ } q^{3}}{1-q^{3}} \sin 3 x+\frac{\sqrt{ } q^{5}}{1-q^{5}} \sin 5 x+\ldots .
$$

(8.) $\quad \sqrt{ } q\left\{\frac{\left(1-q^{2}\right)\left(1-q^{4}\right) \ldots}{(1+q)\left(1+q^{3}\right) \ldots}\right\}^{2} \frac{\cos \left(1+2 q^{2} \cos 2 x+q^{4}\right)\left(1+2 q^{4} \cos 2 x+q^{8}\right) \ldots}{\left(1-2 q \cos 2 x+q^{2}\right)\left(1-2 q^{3} \cos 2 x+q^{6}\right) \ldots}$

$$
=\frac{\sqrt{ } q}{1+q} \cos x+\frac{\sqrt{ } q^{3}}{1+q^{3}} \cos 3 x+\frac{\sqrt{ } q^{5}}{1+q^{5}} \cos 5 x+\ldots . .
$$

(9.)

$$
\left\{\frac{\left(1-q^{2}\right)\left(1-q^{4}\right) \ldots}{\left(1+q^{2}\right)\left(1+q^{4}\right) \ldots}\right\}^{2} \frac{\left(1+2 q \cos 2 x+q^{2}\right)\left(1+2 q^{3} \cos 2 x+q^{6}\right) \ldots}{\left(1-2 q \cos 2 x+q^{2}\right)\left(1-2 q^{3} \cos 2 x+q^{6}\right) \ldots}
$$

$$
=1+\frac{4 q}{1+q^{2}} \cos 2 x+\frac{4 q^{2}}{1+q^{4}} \cos 4 x+\frac{4 q^{3}}{1+q^{6}} \cos 6 x+\ldots,
$$

(10.) $\quad \sqrt{ } q\left\{\frac{\left(1-q^{2}\right)\left(1-q^{4}\right) \ldots}{(1-q)\left(1-q^{3}\right) \ldots}\right\}^{2} \frac{\left(1-2 q^{2} \cos 2 x+q^{4}\right)\left(1-2 q^{4} \cos 2 x+q^{8}\right) \ldots}{\left(1-2 q \cos 2 x+q^{2}\right)\left(1-2 q^{3} \cos 2 x+q^{6}\right) \ldots}$

$$
=\frac{\sqrt{ } q \cdot(1+q)}{1-2 q \cos 2 x+q^{2}}+\frac{\sqrt{ } q^{3} \cdot\left(1+q^{3}\right)}{1-2 q^{3} \cos 2 x+q^{6}}+\frac{\sqrt{ } q^{5} \cdot\left(1+q^{5}\right)}{1-2 q^{5} \cos 2 x+q^{10}}+\ldots,
$$

$$
\begin{aligned}
& \sqrt{ } q\left\{\frac{\left(1-q^{2}\right)\left(1-q^{4}\right) \ldots .}{(1+q)\left(1+q^{3}\right) \ldots}\right\}^{2} \frac{\left(1+2 q^{2} \cos 2 x+q^{4}\right)\left(1+2 q^{4} \cos 2 x+q^{8}\right) \ldots}{\left(1-2 q \cos 2 x+q^{2}\right)\left(1-2 q^{3} \cos 2 x+q^{6}\right) \ldots} \\
= & \frac{\sqrt{ } q \cdot(1-q)}{1-2 q \cos 2 x+q^{2}}-\frac{\dot{V} q^{3} \cdot\left(1-q^{3}\right)}{1-2 q^{3} \cos 2 x+q^{6}}+\frac{\sqrt{ } q^{5} \cdot\left(1-q^{5}\right)}{1-2 q^{5} \cos 2 x+q^{10}}-\ldots
\end{aligned}
$$

Aus (3.) folgt nämlich (4.) für $\alpha=\infty$, und (6.) für $\alpha=1$. Hierdurch enlsteht zunächst:

$$
\begin{gathered}
\left\{\frac{\left(1-q^{2}\right)\left(1-q^{4}\right) \ldots . .}{(1-q)\left(1-q^{3}\right) \ldots}\right\}^{2} \frac{\left(1-2 q \cos 2 x+q^{2}\right)\left(1-2 q^{3} \cos 2 x+q^{6}\right) \ldots}{\left(1-2 q^{2} \cos 2 x+q^{4}\right)\left(1-2 q^{4} \cos 2 x+q^{8}\right) \ldots} \\
=\frac{\left(1-q^{2}\right)}{(1-q)^{2}}-\frac{2\left(1-q^{2}\right)}{(1-q)\left(1-q^{3}\right)} q \cos 2 x-\frac{2\left(1-q^{2}\right)}{\left(1-q^{3}\right)\left(1-q^{5}\right)} q^{3} \cos 4 x-\ldots
\end{gathered}
$$

Dividirt man beide Seiten dieser Gleichung durch $\sin x$, entwickelt rechts nach Sinus der Vielfachen von $x$, mit Hülfe der Formel

$$
\begin{aligned}
\frac{\cos 2 m x}{\sin x} & =\frac{\cos (2 m-2) x}{\sin x}-2 \sin (2 m-1) x \\
& =\frac{1}{\sin x}-2 \sin x-2 \sin 3 x-\ldots-2 \sin (2 m-1) x,
\end{aligned}
$$

und vereinfacht dann die Coëfficienten der Sinus der Vielfachen durch die Zerlegung

$$
\frac{q^{2 p-1}\left(1-q^{2}\right)}{\left(1-q^{2 p-1}\right)\left(1-q^{2 p+1}\right)}=\frac{q^{2 p-1}}{1-q^{2 p-1}}-\frac{q^{2 p+1}}{1-q^{2 p+1}},
$$


so erhält man die Gleichung (6.) in der oben aufgestellten Form; ferner (9.) aus (3.) für $\alpha=\frac{\pi i}{\log q}$, nach Vertauschung von $x$ mit $\frac{1}{2} \pi-x$. Aus (4.) entsteht (5.), und (7.) aus (6.), wenn man $x-\frac{1}{2}(i \log q)$ für $x$ setzt, und (8.) aus (7.) durch gleichzeitige Vertauschung von $q$ mit $-q$ und $x$ mit $\frac{1}{2} \pi-x$. Ferner erhält man (10.) aus (7.), wenn man die identische Gleichung

$$
\frac{1}{1-\xi}+\frac{\zeta}{1-q^{2} \xi}+\frac{\zeta^{2}}{1-q^{4} \xi}+\ldots=\frac{1}{1-\zeta}+\frac{\xi}{1-q^{2} \zeta}+\frac{\xi^{2}}{1-q^{4} \zeta}+\ldots
$$

mit $\sqrt{\zeta}$ multiplicirt, und darauf $\xi=q$ und $\zeta$ einmal gleich $q e^{2 i x}$, dann gleich $q e^{-2 i x}$ setzt, und hierauf die eine der so entstehenden Gleichungen von der andern subtrahirt. Hätte man $\xi=-q$ gemacht, so hätte sich (11.) aus (8.) ergeben.

Die Formeln, welche aus (4.) bis (11.) für gewisse einfache Werthe von $x$, z. B. $x=0, \frac{1}{2} \pi, \pi$, folgen, wird man den obigen leicht hinzufügen.

Aufser den Gleichungen (4.) bis (11.), die sich aus (3.) unmittelbar ergeben, folgen aus dieser allgemeinen Formel noch einige andere interessante Beziehungen, die hier Platz finden mögen, obgleich sie nicht unmittelbar zu unserm Zwecke nothwendig sind.

A) Um die bekannte Gleichung

$$
\left\{1+2 q^{1}+2 q^{4}+2 q^{9}+\ldots .\right\}^{2}=1+\frac{4 q}{1-q}-\frac{4 q^{3}}{1-\eta^{3}}+\ldots
$$

zu beweisen, mache man $x=\frac{1}{2} \pi$ in (4.) und (6.); man hat dann nur noch zu zeigen, dafs

$\left\{\left(1-q^{2}\right)\left(1-q^{4}\right) \ldots\right\}^{2}\left\{(1+q)\left(1+q^{3}\right) \ldots\right\}^{4}=\left\{\frac{\left(1-q^{2}\right)\left(1-q^{4}\right) \ldots}{(1-q)\left(1-q^{3}\right) \ldots}\right\}^{2}\left\{\frac{(1+q)\left(1+q^{3}\right) \ldots}{\left(1+q^{2}\right)\left(1+q^{4}\right) \ldots}\right\}^{2}$ oder dafs

$$
(1+q)\left(1+q^{2}\right)\left(1+q^{3}\right) \ldots=\frac{1}{(1-q)\left(1-q^{3}\right)\left(1-q^{5}\right) \ldots} \text { ist. }
$$

Diese Gleichung verificirt man auf der Stelle, wenn man sie mit $(1-q)\left(1-q^{2}\right)\left(1-q^{3}\right) \ldots$ multiplicirt, wodurch die Identität entsteht:

$\left\{(1+q)\left(1+q^{2}\right)\left(1+q^{3}\right) \ldots.\right\}\left\{(1-q)\left(1-q^{2}\right)\left(1-q^{3}\right) \ldots\right\}=\left(1-q^{2}\right)\left(1-q^{4}\right)\left(1-q^{6}\right) \ldots \ldots$

B) Die so eben hergeleitete Formel ist nur ein specieller Fall einer weit allgemeinern, die aus der identischen Gleichung folgt:

$$
\begin{gathered}
\left\{\left(1-2 q \cos 2 x+q^{2}\right)\left(1-2 q^{3} \cos 2 x+q^{6}\right) \ldots\right\}^{2} \\
\left.=\frac{\left(1-2 q \cos 2 x+q^{2}\right)\left(1-2 q^{3} \cos 2 x+q^{6}\right) \ldots .}{\sin x\left(1-2 q^{2} \cos 2 x+q^{4}\right)\left(1-2 q^{4} \cos 2 x+q^{8}\right) \ldots .}\right\} \\
\quad\left\{\sin x\left(1-2 q \cos 2 x+q^{2}\right)\left(1-2 q^{2} \cos 2 x+q^{4}\right)\left(1-2 q^{3} \cos 2 x+q^{6}\right) \ldots\right\} .
\end{gathered}
$$


Setzt man für die drei in Parenthesen eingeschlossenen unendlichen Producte ihre Werthe aus (4.), (6.) und (5.), nachdem man in letzterer $q$ für $q^{2}$ geschrieben hat, benutzt endlich auch die Formel

$$
\frac{(1-q)\left(1-q^{2}\right)\left(1-q^{3}\right) \ldots}{\left\{(1-q)\left(1-q^{3}\right)\left(1-q^{5}\right) \ldots\right\}^{2}}=1+\frac{1.2}{q^{2}}+\frac{2.3}{q^{2}}+q^{\frac{3.4}{2}}+\ldots .
$$

die sich aus (5.) durch Verwandlung von $q^{2}$ und $x$ in $q$ und $\frac{1}{2} \pi$ ergiebt, so erhält man die Gleichung

$$
\left.\begin{array}{rl}
\left\{1-2 q^{1} \cos 2 x+2 q^{4} \cos 4 x-2 q^{9} \cos 6 x+\ldots\right\}^{2} \\
= & \left\{\frac{1}{\sin x}+\frac{4 q}{1-q} \sin x+\frac{4 q^{3}}{1-q^{3}} \sin 3 x+\ldots\right\} \\
& \left\{\frac{\sin x-\frac{1.2}{q^{2}} \sin 3 x+q^{2} \sin 5 x-\frac{q^{2}}{\eta^{2}} \sin 7 x+\ldots .}{1+\frac{12}{q^{2}}}+q^{\frac{2.3}{2}}+\frac{3.4}{q^{2}}+\ldots .\right.
\end{array}\right\}
$$

welche für $x=\frac{1}{2} \pi$ in die unter $(\boldsymbol{A})$ abgeleitete übergeht.

$C$ ) Endlich selzen wir für $\alpha$ eine gerade Zahl $\alpha=2 m$ in (3.), und finden so die Reihen-Entwickelung eines endlichen Products

$$
\left(1-2 q \cos 2 x+q^{2}\right)\left(1-2 q^{3} \cos 2 x+q^{6}\right) \ldots\left(1-2 q^{2 m-1} \cos 2 x+q^{4 m-2}\right)
$$

nach Cosinus der Vielfachen von $x$. Das Product auf der rechten Seite von (3.), welches noch mit der unendlichen Reihe zu multipliciren ist, verwandelt sich dadurch in

$$
\begin{gathered}
\frac{\left\{\left(1-q^{2 m+2}\right)\left(1-q^{2 m+4}\right)\left(1-q^{2 m+6}\right) \ldots\right\}^{2}}{\left\{\left(1-q^{2}\right)\left(1-q^{4}\right) \ldots\right\}\left\{\left(1-q^{4 m+2}\right)\left(1-q^{4 m+4}\right) \ldots\right\}}=\frac{\left(1-q^{2 m+2}\right)\left(1-q^{2 m+4}\right) \ldots\left(1-q^{4 m}\right)}{\left(1-q^{2}\right)\left(1-q^{4}\right) \ldots\left(1-q^{2 m}\right)} \\
=\frac{\left(1-q^{2}\right)\left(1-q^{4}\right) \ldots\left(1-q^{4 m}\right)}{\left\{\left(1-q^{2}\right)\left(1-q^{4}\right) \ldots\left(1-q^{2 m}\right)\right\}^{2}} \\
=\frac{(1+q)\left(1+q^{2}\right) \ldots\left(1+q^{2 m}\right)}{(1-q)\left(1-q^{2}\right) \ldots .\left(1-q^{2 m}\right)}\left\{(1-q)\left(1-q^{3}\right) \ldots\left(1-q^{2 m-1}\right)\right\}^{2} .
\end{gathered}
$$

So findet man

$$
\begin{gathered}
\frac{(1-q)\left(1-q^{2}\right) \ldots\left(1-q^{2 m}\right)}{(1+q)\left(1+q^{2}\right) \ldots\left(1+q^{2 m}\right)} \cdot \frac{\left(1-2 q \cos 2 . x+q^{2}\right)\left(1-2 q^{3} \cos 2 x+q^{6}\right) \ldots\left(1-2 q^{2 m-1} \cos 2 x+q^{4 m-2}\right)}{\left\{(1-q)\left(1-q^{3}\right) \ldots\left(1-q^{2 m-1}\right)\right\}^{2}} \\
=1-\frac{2\left(1-q^{2 m}\right)}{\left(1-q^{2 m+2}\right)} q^{1} \cos 2 x+\frac{2\left(1-q^{2 m}\right)\left(1-q^{2 m-2}\right)}{\left(1-q^{2 m+2}\right)\left(1-q^{2 m+4}\right)} q^{4} \cos 4 x \\
-\frac{2\left(1-q^{2 m}\right)\left(1-q^{2 m-2}\right)\left(1-q^{2 m-4}\right)}{\left(1-q^{2 m+2}\right)\left(1-q^{2 m+4}\right)\left(1-q^{2 m+6}\right)} q^{9} \cos 6 x+\ldots .,
\end{gathered}
$$

Für $x=0$ erhält man die Entwickelung des endlichen Products

$$
\frac{(1-q)\left(1-q^{2}\right) \ldots\left(1-q^{2 m}\right)}{(1+q)\left(1+q^{2}\right) \ldots\left(1+q^{2 m}\right)}
$$


in eine Reihe, die für $m==\infty$ in die bekannte

übergeht.

$$
1-2 q^{1}+2 q^{4}-2 q^{9}+\ldots
$$

II. A b s c h n i t t.

Die drei Reihen (7.), (8.), (9.)

$$
\begin{aligned}
& \text { (a.) } \frac{1}{2 i} \sum_{m=-\infty}^{m=\infty} \frac{q^{\frac{1}{2}(2 m+1)}}{1-q^{2 m+1}} z^{\frac{1}{2}(2 m+1)}, \\
& \text { (b.) } \frac{1}{2} \sum_{m=-\infty}^{m=\infty} \frac{q^{\frac{1}{2}(2 m+1)}}{1+q^{2 m+1}} z^{\frac{1}{2(2 m+1)}}, \\
& \text { (c.) } 2 \sum_{m=-\infty}^{m=\infty} \frac{q^{m}}{1+q^{2 m}} z^{m},
\end{aligned}
$$

deren Ausdrücke durch unendliche Producte im vorigen Abschnitt gefunden wurden, sollen hier zu je zweien mit einander multiplicirt werden. Bezeichnet man ihre Quadrate mit $(\boldsymbol{a})^{2},(\boldsymbol{b})^{2},(\boldsymbol{c})^{2}$, so ist in $(\boldsymbol{a})^{2}$ mit $-\frac{1}{4} \boldsymbol{z}^{m}$ die Reihe

$$
\begin{aligned}
& \left\{\frac{q^{m}}{\left(1-q^{2 m+1}\right)\left(1-q^{-1}\right)}+\ldots+\frac{q^{m}}{\left(1-q^{2 m+2 p+1}\right)\left(1-q^{-2 p-1}\right)}+\ldots\right\} \\
+ & \left\{\frac{q^{m}}{\left(1-q^{2 m-1}\right)(1-q)}+\ldots .+\frac{q^{m}}{\left(1-q^{2 m-2 p-1}\right)\left(1-q^{2 p+1}\right)}+\ldots\right\}
\end{aligned}
$$

multiplicirt. Die Glieder der in den beiden Parenthesen befindlichen Reihen lassen sich leicht zerlegen, und zwar setze ich für das pte Glied in der ersten:

$$
\frac{q^{m}}{1-q^{2 m}}\left\{-\frac{q^{2 p+1}}{1-q^{2 p+1}}+\frac{q^{2 m+2 p+1}}{1-q^{2 m+2 p+1}}\right\}
$$

und für das pte Glied der zweiten:

$$
\frac{q^{m}}{1-q^{2 m}}\left\{\frac{q^{2 p+1}}{1-q^{2 p+1}}+\frac{1}{1-q^{2 m-2 p-1}}\right\} \text {. }
$$

Nun ist der Coëfficient von $-\frac{1}{4} z^{m}$ die Summe (Differenz) von vier Reihen *),

*) Die Zerlegung der pten Glieder, die auf verschiedene Arten Statt finden konnte, war deshalb nicht ganz willkürlich, weil, nachdem sie geschehn ist, die durch Zerlegung eines Gliedes entstehenden beiden nicht zusammenbleiben sollen, sondern gegeneinander verschoben werden. Es müssen also solche Zerlegungen vorgenommen werden, dafs die neuen Reihen nicht durch die Vorzeichen convergiren, wie dieses mit der Reihe $1-\frac{1}{2}+\frac{1}{3}-\frac{1}{4}+\frac{1}{5}-\ldots$ der Fall ist, sondern unabhängig von diesen. So convergirt $z$. B. die Reihe, deren $p$ tes Glied $\frac{q^{2 p+1}}{1-q^{2 p+1}}$ oder $\frac{1}{1-q^{2 m-2 p-1}}$ ist, während eine Reihe, deren 
von denen zwei sich sofort heben, also gleich

$$
\frac{q^{m}}{\left(1-q^{2 m}\right)}\left\{\sum_{p=0}^{p=\infty} \frac{q^{2 m+2 p+1}}{1-q^{2 m+2 p+1}}+\sum_{p=0}^{p=\infty} \frac{1}{1-q^{2 m-2 p-1}}\right\} \text {. }
$$

Die letzte Summe zerfällt in eine von $p=0$ bis $p=m-1$, eine zweite von $m$ bis $2 m-1$, und eine dritte von $2 m$ bis $\infty$. Beachtet man, dafs $\frac{1}{1-q^{-r}}=-\frac{q^{r}}{1-q^{r}}$, so findet man für obigen Ausdruck:

$$
\begin{gathered}
\frac{q^{m}}{1-q^{2 m}}\left\{\frac{1}{1-q^{2 m-1}}+\frac{1}{1-q^{2 m-3}}+\cdots+\frac{1}{1-q^{-1}}-\frac{q}{1-q^{1}}-\ldots-\frac{q^{2 m-1}}{1-q^{2 m-1}}\right\} \\
=\frac{m q^{m}}{1-q^{2 m}}
\end{gathered}
$$

mit Ausnahme des Werthes $\boldsymbol{m}=\mathbf{0}$.

So erhält man:

$$
\begin{aligned}
2(\boldsymbol{a})^{2}= & \left\{\frac{q}{(1-q)^{2}}+\frac{q^{3}}{\left(1-q^{3}\right)^{2}}+\frac{q^{5}}{\left(1-q^{5}\right)^{2}}+\ldots\right\} \\
& -\frac{1 \cdot q}{1-q^{2}} \cos 2 x-\frac{2 \cdot q^{2}}{1-q^{4}} \cos 4 x-\frac{3 \cdot q^{3}}{1-q^{6}} \cos 6 x-\ldots,
\end{aligned}
$$

und durch einfache Vertauschungen:

$$
\begin{aligned}
2(b)^{2}= & \left\{\frac{q}{(1+q)^{2}}+\frac{q^{3}}{\left(1+q^{3}\right)^{2}}+\frac{q^{5}}{\left(1+q^{5}\right)^{2}}+\ldots\right\} \\
& +\frac{1 \cdot q}{1-q^{2}} \cos 2 x+\frac{2 \cdot q^{2}}{1-q^{4}} \cos 4 x+\frac{3 \cdot q^{3}}{1-q^{6}} \cos 6 x+\ldots \ldots
\end{aligned}
$$

Auf ähnliche Art wie (12.) ergiebt sich

$$
\begin{aligned}
\frac{1}{8}(c)^{2}= & \left\{\frac{1}{8}+\frac{q^{2}}{\left(1+q^{2}\right)^{2}}+\frac{q^{4}}{\left(1+q^{4}\right)^{2}}+\ldots\right\} \\
& +\frac{1 \cdot q}{1-q^{2}} \cos 2 x+\frac{2 \cdot q^{2}}{1-q^{4}} \cos 4 x+\frac{3 \cdot q^{3}}{1-q^{6}} \cos 6 x+\ldots \ldots
\end{aligned}
$$

Ehe wir zur Betrachtung der noch fehlenden Producte $(\boldsymbol{a})(\boldsymbol{b}),(\boldsymbol{a})(\boldsymbol{c}),(\boldsymbol{b})(\boldsymbol{c})$ übergehn, wollen wir aus den so eben gefundenen Formeln einige Folgerungen ziehen. Um unsere Resultate in der bekannten Bezeichnungsweise auszudrücken, führen wir drei Gröfsen $k, k^{\prime}, \boldsymbol{K}$ ein, indem wir setzen

$p$ tes Glied $\frac{1}{1-q^{2 p+1}}$ ist, divergiren würde. Deshalb durfte z. B. das $p$ te Glied der zweiten Reihe nicht so dargestellt werden:

$$
\frac{q^{m}}{1-q^{2 m}}\left\{\frac{1}{1-q^{2 p+1}}+\frac{q^{2 m-2 p-1}}{1-q^{2 m-2 p-1}}\right\} \text {. }
$$

Der hier erwähnte Umstand ist auch bei allen folgenden Zerlegungen sorgfältig zu beachten. 


$$
\begin{aligned}
\text { (15.) } \quad k & =4\urcorner q\left\{\frac{\left(1+q^{2}\right)\left(1+q^{4}\right) \ldots .}{(1+q)\left(1+q^{3}\right) \ldots .}\right\}^{4}, \\
\text { (16.) } \quad k & =\left\{\frac{(1-q)\left(1-q^{3}\right)\left(1-q^{5}\right) \ldots}{(1+q)\left(1+q^{3}\right)\left(1+q^{5}\right) \ldots}\right\}^{4}, \\
\text { (17.) } \quad \frac{2 l k K}{\pi} & =4 \sqrt{ } q\left\{\frac{\left(1-q^{4}\right)\left(1-q^{8}\right)\left(1-q^{12}\right) \ldots}{\left(1-q^{2}\right)\left(1-q^{6}\right)\left(1-q^{10}\right) \ldots}\right\}^{2} .
\end{aligned}
$$

Dafs $k$ mit $k^{\prime}$ durch die Gleichung $k^{2}+k^{\prime 2}=1$ zusammenhängt, wird sich im weitern Verlaufe leicht ergeben; hier wird diese Relation nicht vorausgesetzt.

Aus (12.) und (13.) erhellt, dafs $(a)^{2}+(b)^{2}$ von $x$ unabhängig ist, so dafs, wenn $g$ einen von $x$ unabhängigen Werth darstellt, nämlich

$$
2 g^{2}=\frac{q}{(1-q)^{2}}+\frac{q}{(1+q)^{2}}+\frac{q^{3}}{\left(1-q^{3}\right)^{2}}+\frac{q^{3}}{\left(1+q^{3}\right)^{2}}+\ldots,
$$

(a) gleich $g$ mal einem Sinus, (b) gleich $g$ mal dem Cosinus desselben Winkels geselzt werden kann, also

$$
\begin{aligned}
& (\boldsymbol{a})=g \sin f(x), \\
& (b)=g \cos f(x) .
\end{aligned}
$$

Der Werth der Constante $g$ läfst sich übrigens leicht als unendliches Product darstellen, indem für jedes $x$, also auch für $x=0, g=l\left((a)^{2}+(b)^{2}\right)$ wird. Da nun für $x=0,(a)$ verschwindet und (b) nach (8.) und (17.) gleich $\frac{k K}{2 \pi}$ ist, so wird

$$
\begin{aligned}
& \text { (18.) } \quad(a)=\frac{l K}{2 \pi} \sin f(x), \\
& \text { (19.) } \quad(b)=\frac{l k K}{2 \pi} \cos f(x) .
\end{aligned}
$$

Ferner findet man durch Vergleichung von (12.) und (14.)

$$
(4 a)^{2}+(c)^{2}=h^{2} \text {, }
$$

wo $h$ eine Constante (nach $x$ ) bezeichnet, die sich wiederum durch ein unendliches Product darstellen läfst, indem für $x$ ein besonderer Werth, $x=0$, gesetzt wird. Dann hat man $(a)=0$; ferner $(c)$ nach (9.), (15.) und (17.) gleich $\frac{2 K}{\pi}$, also $h=\frac{2 K}{\pi}$, folglich nach (18.)

$$
(c)=\frac{2 K}{\pi} \sqrt{ }\left(1-k^{2} \sin ^{2} f(x)\right),
$$

und macht man zur Abkürzung

$$
\Delta f(x)=\sqrt{ }\left(1-k^{2} \sin ^{2} f(x)\right),
$$

Crelle's Journal f. d. M. Bd. XXXIX. Heft 2 
so ist

$$
\left(20^{*} .\right) \quad(c)=\frac{2 K}{\pi} \Delta f(x) .
$$

Da nun für $x=\frac{1}{2} \pi$ nach (7.) und (17.), (a) sich in $\frac{k K}{2 \pi},(c)$ nach (9.), (15.), (16.) und (17.) sich in $\frac{2 K k^{\prime}}{\pi}$ verwandelt, so wird nach (18.)

$$
\sin f\left(\frac{1}{2} \pi\right)=1
$$

und nach (20.)

$$
\frac{2 K k^{\prime}}{\pi}=\frac{2 K}{\pi} \sqrt{ }\left(1-k^{2} \sin ^{2} f\left(\frac{1}{2} \pi\right)\right)=\frac{2 K}{\pi} \sqrt{ }\left(1-k^{2}\right),
$$

also

$$
\text { (21.) } \quad k^{2}+k^{\prime 2}=1 \text {. }
$$

Wir haben nun die drei Gleichungen

$$
\left\{\begin{array}{l}
\frac{k K}{2 \pi} \sin (f x)=\frac{\sqrt{ } q}{1-q} \sin x+\frac{\sqrt{ } q^{3}}{1-q^{3}} \sin 3 x+\ldots, \\
\frac{k K}{2 \pi} \cos (f x)=\frac{\sqrt{ } q}{1+q} \cos x+\frac{\sqrt{ } q^{3}}{1+q^{3}} \cos 3 x+\ldots, \\
\frac{2 K}{\pi} \Delta f(x)=1+\frac{4 q}{1+q^{2}} \cos 2 x+\frac{4 q^{2}}{1+q^{4}} \cos 4 x+\ldots
\end{array}\right.
$$

bewiesen, die man noch mit (6.) bis (11.) und ähnlichen Formeln combiniren kann.

Von den übrigen Verbindungen $(\boldsymbol{a})(\boldsymbol{b}),(\boldsymbol{a})(\boldsymbol{c}),(\boldsymbol{b})(\boldsymbol{c})$ wollen wir nur eine bilden, indem die beiden andern nichts geben, was nicht schon aus einer hervorginge. In dem Producte $(b)(c)$ ist, mit $z^{2 m+1}$ multiplicirt:

$$
\begin{aligned}
\frac{1}{2} \frac{q^{\frac{1}{2}(2 m+1)}}{\left(1+q^{2 m+1}\right)} & +\frac{q^{-1}}{\left(1+q^{-2}\right)} \frac{q^{\frac{1}{(2(2 m+3)}}}{\left(1+q^{2 m+3}\right)}+\ldots+\frac{q^{-p}}{\left(1+q^{-2 p}\right)} \frac{q^{\frac{1}{2}(2 m+2 p+1)}}{\left(1+q^{2 m+2 p+1}\right)}+\ldots \\
& +\frac{q^{1}}{\left(1+q^{2}\right)} \frac{q^{\frac{1}{(2 m-1)}}}{\left(1+q^{2 m-1}\right)}+\ldots+\frac{q^{p}}{\left(1+q^{2 p}\right)} \frac{q^{\frac{2}{(2 m-2 p+1)}}}{\left(1+q^{2 m-2 p+1}\right)}+\ldots \ldots
\end{aligned}
$$

Für das pte Glied der ersten Reihe setze man

$$
\frac{q^{\frac{1}{2}(2 m+1)}}{1-q^{2 m+1}}\left\{\frac{q^{2 p}}{1+q^{2 \rho}}-\frac{q^{2 m+2 p+1}}{1+q^{2 m+2 p+1}}\right\}
$$

für das pte Glied der zweiten

$$
\frac{q^{2}(2 m+1)}{1-q^{2 m+1}}\left\{-\frac{q^{2 p}}{1+q^{2 p}}+\frac{1}{1+q^{2 m-2 p+1}}\right\},
$$

so dafs der Coëfficient von $z^{2 m+1}$ (indem man in der oberen Reihe von $p=0$, in der untern von $p=1$ an summiren mufs), 


$$
\begin{gathered}
\frac{q^{\frac{1}{2}(2 m+1)}}{1-q^{2 m+1}}\left\{\frac{1}{2}+\sum_{p=1}^{p=2 m} \frac{1}{1+q^{2 m-2 p+1}}+\sum_{p=2 m+1}^{p=\infty} \frac{1}{1+q^{2 m-2 p+1}}-\sum_{p=0}^{p=\infty} \frac{q^{2 m+2 p+1}}{1+q^{2 m+2 p+1}}\right\} \\
=\left(m+\frac{1}{2}\right) \frac{q^{1}(2 m+1)}{1-q^{2 m+1}}
\end{gathered}
$$

wird. Man hat also nach (19.) und (20*.):

$$
\begin{aligned}
\frac{k K^{2}}{\pi^{2}} \cos f(x) \Delta f(x) & =\frac{1 \cdot q^{\frac{1}{2}}}{1-q} \cos x+\frac{3 \cdot q^{\frac{3}{2}}}{1-q^{3}} \cos 3 x+\frac{5 \cdot q^{\frac{5}{2}}}{1-q^{5}} \cos 5 x+\ldots \\
& =\frac{d}{d x}\left\{\frac{q^{\frac{1}{2}}}{1-q} \sin x+\frac{q^{\frac{3}{2}}}{1-q^{3}} \sin 3 x+\frac{q^{\frac{5}{2}}}{1-q^{5}} \sin 5 x+\ldots\right\} \\
& =\frac{l K}{2 \pi} \frac{d \sin f(x)}{d x},
\end{aligned}
$$

folglich

$$
\frac{2 K x}{\pi}=\int \frac{d \sin f(x)}{\cos f(x) \Delta f(x)},
$$

und da $\sin f(x)$ nach (22.) für $x=0$ verschwindet, so ist das Integral von $\sin f(x)=0$ anzunehmen. Es genügt demnach

der Gleichung

$$
z=\sin f(x)=\frac{2 \pi}{i K}\left\{\frac{\sqrt{ } q}{1-q} \sin x+\frac{\sqrt{ } q^{3}}{1-q^{3}} \sin 3 x+\ldots\right\}
$$

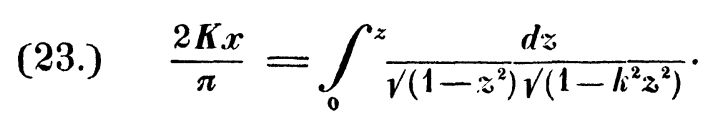

\section{A b s c h n i t t.}

Während wir im Vorhergehenden zwei von den Reihen (a.), (b.), (c.) mit einander verbanden, die dasselbe Argument $x$ hatten, wollen wir jetzt das Product dreier von ihnen betrachten, von denen die eine das Argument $\boldsymbol{x}$ hat, die zweite $y$, die dritte das aus beiden symmetrisch zusammengesetzte $x+y$. Setzt man deshalb nicht nur $z=e^{2 x i}$, sondern auch $v=e^{2 y i}$, so wird z. B. in dem Producte

$$
\left\{\frac{1}{2 i} \sum_{m=-\infty}^{m=\infty} \frac{q^{\frac{1}{(2 m+1)}}}{1-q^{2 m+1}} z^{\frac{1}{2}(2 m+1)}\right\}\left\{\left\{\frac{1}{2} \sum_{n=-\infty}^{n=\infty} \frac{q^{1}(2 n+1)}{1+q^{2 n+1}} z^{\frac{1}{2}(2 n+1)}\right\}\left\{2 \sum_{r=-\infty}^{r=\infty} \frac{q^{r}}{1+q^{2 r}}(\boldsymbol{z} v)^{r}\right\}\right.
$$

mit $\frac{1}{2 i} z^{\frac{1}{2(2 m+1)}} v^{\frac{1}{2}(2 n+1)}$ folgende Reihe multiplicirt sein :

(24.)

$$
\begin{aligned}
& \left\{\frac{1}{2} \cdot \frac{q^{\frac{3}{(2 m+1)}}}{\left(1-q^{2} m+1\right)} \frac{q^{\frac{1}{2}(2 n+1)}}{\left(1+q^{2 n+1}\right)}+\ldots \ldots \ldots+\frac{q^{\frac{1}{2}(2 m+2 p+1)}}{\left(1-q^{2 m+2 p+1}\right)} \frac{q^{\frac{1}{(2 n+2 p+1}}}{\left(1+q^{2 n+2 p+1}\right)} \frac{q^{-p}}{\left(1+q^{-2 p}\right)}+\ldots\right\} \\
& +\left\{\frac{q^{\frac{1}{2}(2 m-1)}}{\left(1-q^{2 m-1}\right)} \frac{q^{\frac{1}{2}(2 n-1)}}{\left(1+q^{2 n-1}\right)} \frac{q^{1}}{\left(1+q^{2}\right)}+\ldots .+\frac{q^{\frac{1}{(2 m-2 p+1)}}}{\left(1-q^{2 m-2 p+1}\right)} \frac{q^{\frac{1}{(2 n-2 p+1)}}}{\left(1+q^{2 n-2 p+1}\right)} \frac{q^{p}}{\left(1+q^{2 p}\right)}+\ldots . .\right.
\end{aligned}
$$


Das pte Glied der obern Reihe läfst sich zunächst als die Summe zweier darstellen; es ist nämlich gleich

$$
\frac{q^{m+n+1}}{1+q^{2 m+1}}\left\{\frac{q^{p}}{\left(1+q^{2 n+2 p+1}\right)\left(1+q^{-2 \prime^{\prime}}\right)}+\frac{q^{3 p+2 m+1}}{\left(1+q^{2 n+2 p+1}\right)\left(1-q^{2 m+2 p+1}\right)}\right\},
$$

und das erste Glied der in der vorstehenden Parenthese befindlichen wird gleich

das zweite gleich

$$
\frac{1}{1-q^{2 n+1}}\left\{\frac{q^{p}}{1+q^{2 n+2 p+1}}-\frac{q^{p}}{1+q^{2 \rho}}\right\}
$$

$$
\frac{1}{1+q^{2 n-2 n}}\left\{\frac{q^{p}}{1-q^{2 m+2 p+1}}-\frac{q^{p}}{1+q^{2 n+2 p+1}}\right\}
$$

so dafs wir für die erste Reihe von (24.)

$$
\begin{aligned}
& \frac{q^{\frac{1}{2}(2 m+1)}}{1+q^{2 n+1}}\left\{\frac{q^{1}(2 n+1)}{1-q^{2 n+1}}\left\{\sum_{p=0}^{p=\infty} \frac{q^{p}}{1+q^{2 n+2 p+1}}-\sum_{p=0}^{p=\infty} \frac{q^{p}}{1+q^{2 p}}\right\}\right. \\
& \left.+\frac{q^{1}(2 n+1)}{1+q^{2 n-2 m}}\left\{\sum_{p=0}^{p=\infty} \frac{q^{p}}{1-q^{2 m+2 p+1}}-\sum_{p=0}^{p=\infty} \frac{q^{p}}{1+q^{2 n+2 p+1}}\right\}\right\}
\end{aligned}
$$

erhalten. Wird die Summation nach $\boldsymbol{p}$ in der obigen Formel nicht mehr von 0 bis $\infty$, sondern von -1 bis $-\infty$ ausgeführt, so entsteht die zweite Reihe des Ausdrucks (24.), und der ganze Coëfficient von $\frac{1}{2 i} \mathbf{z}^{\frac{1}{l^{(2 m+1)}}} v^{\frac{1}{2}(2 n+1)}$ wird

$$
-\frac{q^{\frac{1}{2}(2 m+1)}}{\left(1+q^{2 m+1}\right)} \frac{q^{\frac{1}{2}(2 n+1)}}{\left(1-q^{2 n+1}\right)} \sum_{p=-\infty}^{p=\infty} \frac{q^{p}}{1+q^{2 p}}+\frac{q^{\frac{1}{2}(2 n+1)}}{\left(1-q^{2 n+1}\right)} \frac{q^{n-m}}{\left(1+q^{2 n-2 m}\right)} \sum_{p=-\infty}^{p=\infty} \frac{q^{\frac{1}{2}(2 p+1)}}{1-q^{2 p+1}} .
$$

Diese Formel folgt unmittelbar aus der vorhergehenden, da

$$
\sum_{p=-\infty}^{p=\infty} \frac{q^{p}}{1-q^{2 m+2 p+1}}=q^{-\frac{1}{2}(2 m+1)} \sum_{p=-\infty}^{p=\infty} \frac{q^{1}(2 m+2 p+1)}{1-q^{2 m+2 p+1}}
$$

offenbar 0 ist, und

$$
\sum_{p=-\infty}^{p=\infty} \frac{q^{p}}{1+q^{2 n+2 p+1}}=q^{-\frac{1}{2}(2 n+1)} \sum_{p=-\infty}^{p=\infty} \frac{q^{\frac{1}{2}(2 n+2 p+1)}}{1+q^{2 n+2 p+1}}=q^{-\frac{1}{2}(2 n+1)} \sum_{p=-\infty}^{p=\infty} \frac{q^{\frac{1}{2}(2 p+1)}}{1+q^{2 p+1}} .
$$

Setzt man für

$$
\sum_{p=-\infty}^{p=\infty} \frac{q^{n}}{1+q^{2 p}}, \quad \sum_{p=-\infty}^{p=\infty} \frac{q^{1(2 p+1)}}{1+q^{2 p+1}}
$$

noch ihre Werthe $\frac{K}{\pi}$ und $\frac{k K}{\pi}$ aus (22.), so wird in dem Producte der drei mit einander multiplicirten Reihen der Coëfficient von $\frac{1}{2 i} 2^{\frac{1}{(2(2 m+1)}} v^{\frac{1}{3}(2 n+1)}$ gleich

$$
-\frac{K}{\pi} \frac{q^{\frac{1}{(2 n+1)}}}{\left(1+q^{2 n+1}\right)} \frac{q^{\frac{1}{(2 n+1)}}}{\left(1-q^{2 n+1}\right)}+\frac{k K}{\pi} \frac{q^{\frac{1}{(2 n+1)}}}{\left(1-q^{2 n+1}\right)} \frac{q^{n-m}}{\left(1+q^{2 n-2 m}\right)} .
$$


Man hat also

$$
\begin{gathered}
\left(\frac{k K}{2 \pi}\right)^{2} \cdot \frac{2 K}{\pi} \sin f(x) \cos f(y) d f(x+y) \\
=-\frac{K}{\pi} \cdot \frac{k K}{2 \pi} \cdot \frac{k K}{\pi} \cos f(x) \sin f(y)+\frac{k K}{\pi} \cdot \frac{1}{2 i} \sum \frac{q^{12(2 n+1)}}{\left(1-q^{2 n+1}\right)} \frac{q^{n-m}}{\left(1+q^{2 n-2 m}\right)} z^{\frac{1}{2(2 m+1)}} v^{\frac{1}{2(n+1)},},
\end{gathered}
$$

wenn in der letzten Summe, unabhängig von einander, für $\boldsymbol{m}$ und $\boldsymbol{n}$ alle ganze Zahlen von $-\infty$ bis $\infty$ gesetzt werden. Um auch diese unendliche Doppelreihe in ein Product zweier einfachen zu verwandeln, setze man in der Gleichung

$$
\frac{l i K^{2}}{2 \pi^{2}} \sin f(y) \Delta f(x)=\frac{1}{2 i} \sum_{p=-\infty}^{p=\infty}\left\{\frac{q^{p}}{1+q^{2 p}} z^{p} \sum_{n=-\infty}^{n=\infty} \frac{q^{1}(2 n+1)}{1-q^{2 n+1}} v^{\frac{1}{2}(2 n+1)}\right\}
$$

$\boldsymbol{v} \boldsymbol{z}$ statt $v$, während man $z$, also auch $x$, ungeändert läfst. Dadurch ändert sich auf der linken Seite $y$ in $(x+y)$ um, indem

$$
\frac{1}{2 i_{n}} \sum_{n=-\infty}^{n=\infty} \frac{q^{\frac{1}{2}(2 n+1)}}{1-q^{2 n+1}}(z v)^{2 n+1}=\frac{\sqrt[V]{ } q}{1-q} \sin (x+y)+\frac{\sqrt{ } q^{3}}{1-q^{3}} \sin 3(x+y)+\ldots
$$

gleich $\frac{k K}{2 \pi} \sin f(x+y)$ ist, während die rechte Seite sich in

$$
\frac{1}{2 i} \sum \frac{q^{\frac{1}{2}(2 n+1)}}{1-q^{2 n+1}} v^{\frac{1}{2}(2 n+1)} \frac{q^{p}}{1+q^{2 p}} \boldsymbol{2}^{\frac{1}{2}(2 p+2 n+1)}
$$

verwandelt. Macht man $m=\boldsymbol{p}+\boldsymbol{n}$ statt $\boldsymbol{p}$ zum Summationsbuchstaben, und benutzt die Gleichung

so hat man

$$
\frac{q^{m-n}}{1+q^{2 m-2 n}}=\frac{q^{n-m}}{1+q^{2 n-2 m}}
$$

$$
\frac{1}{2 i} \sum_{n=-\infty}^{n=\infty} \frac{q^{\frac{1}{2}(2 n+1)}}{1-q^{2 n+1}} v^{\frac{1}{2}(2 n+1)} \sum_{m=-\infty}^{m=\infty} \frac{q^{n-m}}{1+q^{2 n-2 m}}{z^{\frac{1}{2}(2 m+1)}}^{m}=\frac{l i K^{2}}{2 \pi^{2}} \sin f(x+y) \Delta f(x),
$$

also schliefslich

(25.) $\sin f(x) \cos f(y) \Delta f(x+y)=\sin f(x+y) \Delta f(x)-\cos f(x) \sin f(y)$. Aus dieser Gleichung kann man auch leicht die Ausdrücke von $\Delta \mathrm{am}(\boldsymbol{x}+\boldsymbol{y})$, $\sin \operatorname{am}(x+y)$ und daraus den von $\cos a m(x+y)$ in der gewöhnlichen Form erhalten. Vertauscht man nämlich in (25.) die Gröfsen $x$ und $y$ miteinander, so erhält man

$$
\sin f(y) \cos f(x) \Delta f(x+y)=\sin f(x+y) \Delta f(y)-\cos f(y) \sin f(x)
$$

und hat so zwei Gleichungen, in denen $\sin f(x+y)$ und $\Delta f(x+y)$ linear vorkommen; so dafs man eine von ihnen leicht eliminiren kann. Dadurch findet man 


$$
\begin{aligned}
\Delta f(x+y) & =\frac{\Delta f(x) \Delta f(y)-\sin f(x) \cos f(x) \sin f(y) \cos f(y)}{1-k^{2} \sin ^{2} f^{\prime}(x) \sin ^{2} f^{\prime}(y)}, \\
\sin f(x+y) & =\frac{\sin f(x) \cos f(y) \Delta f(y)+\sin f(y) \cos f(x) \Delta f(x)}{1-k^{2} \sin ^{2} f^{\prime}(x) \sin ^{2} f^{\prime}(y)} .
\end{aligned}
$$

Durch Vertauschung von $q, x$ etc. mit $-q, \frac{1}{2} \pi-x$, etc. oder durch directe Multiplication dreier Reihen erhält man noch andre ähnliche Formeln.

Um noch ein Beispiel der Ausführung einer solchen Multiplication zu geben, betrachte man, obgleich man dadurch kein allgemeineres Resultat als (25.) erhält, das Product

$$
\left(\frac{l K K}{2 \pi}\right)^{2} \frac{2 K}{\pi} \sin f(x) \sin f(y) \Delta f(x+y)=-\frac{1}{2} \Sigma \frac{q^{m+n+r+1} z^{\frac{1}{2}(2 m+1)} v^{\frac{1}{2(2 n+1)}(z v)^{r}}}{\left(1--q^{2 m+1}\right)\left(1-q^{2 n+1}\right)\left(1+q^{2 r}\right)}
$$

die Summation nach $m, n, r$ von $-\infty$ bis $\infty$ ausgeführt. Es ist dann mit $-\frac{1}{2} z^{\frac{1}{2}(2 m+1)} v^{\frac{1}{3}(2 n+1)}$ eine Reihe multiplicirt, deren $p$ tes Glied

$$
\frac{q^{m+n+p+1}}{\left(1-q^{2 m+2 p+1}\right)\left(1-q^{2 n+2 p+1}\right)\left(1+\overline{q^{-2 p}}\right)}
$$

isl, wo $p$ alle ganzen Werthe von $-\infty$ bis $\infty$ annimmt. Dieses Glied zerfällt, so lange nicht $m=n$ ist, in

$$
\begin{aligned}
& \frac{q^{\frac{1}{2}(2 m+1)}}{1+q^{2 m+1}}\left\{\frac{q^{\frac{1}{2}(2 n+1)}}{1+q^{2 n+1}}\left\{-\frac{q^{p}}{1+q^{2 p}}+\frac{q^{p}}{1-q^{2 n+2 p+1}}\right\}\right. \\
& \left.\quad+\frac{q^{\frac{1}{2(2 n+1)}}}{1-q^{2 n-2 m}}\left\{\frac{q^{p}}{1-q^{2 m+2 p+1}}-\frac{q^{p}}{1-q^{2 n+2 p+1}}\right\}\right\}
\end{aligned}
$$

Da die übrigen Glieder bei der Summation von $p=-\infty$ bis $p=\infty$ verschwinden, so reducirt sich der Coëfficient von $-\frac{1}{2} \boldsymbol{s}^{\frac{1}{2}(2 m+1)} v^{\frac{1}{2}(2 n+1)}$ auf

$$
-\frac{q^{\frac{1}{(2 m+1)}}}{\left(1+q^{2 m+1}\right)} \frac{q^{\frac{1}{2}(2 n+1)}}{\left(1+q^{2 n+1}\right)} \sum_{p=-\infty}^{p=\infty} \frac{q^{p}}{1+q^{2 p}}=-\frac{K}{\pi} \cdot \frac{q^{\frac{1}{2}(2 m+1)}}{\left(1+q^{2 m+1}\right)} \frac{q^{\frac{1}{2(2 n+1)}}}{\left(1+q^{2 n+1}\right)} .
$$

Ist aber $m=n$, so wird der Coëfficient von $-\frac{1}{2} z^{\frac{1}{2}(2 m+1)} v^{\frac{1}{2}(2 m+1)}$ gleich der Summe von

$$
\frac{q^{\frac{1}{2}(2 m+1)}}{1+q^{2 m+1}}\left\{\frac{q^{\frac{1}{2}(2 m+1)}}{1+q^{2 m+1}}\left\{-\frac{q^{p}}{1+q^{2 p}}+\frac{q^{p}}{1-q^{2 m+2 p+1}}\right\}+\frac{q^{3 p+3 m+\frac{3}{2}}}{\left(1-q^{2 m+2 p+1}\right)^{2}}\right\}
$$

von $p=-\infty$ bis $p=\infty$, also gleich

$$
-\frac{K}{\pi} \cdot \frac{q^{\frac{1}{2}(2 m+1)}}{\left(1+q^{2 m+1}\right)} \frac{q^{\frac{1}{2}(2 m+1)}}{\left(1+q^{2 m+1}\right)}
$$

vermebrt um $\frac{q^{\frac{1(2 m+1)}{1+q^{2}}}}{1+q^{2 m+1}}$, multiplicirt in die Summe

$$
\sum_{p=-\infty}^{p=\infty} \frac{q^{\frac{3}{2}(2 p+1)}}{\left(1-q^{2 p+1}\right)^{2}}=-\sum_{p=-\infty}^{p=\infty} \frac{q^{\frac{1}{1}(2 p+1)}}{1-q^{2 p+1}}+\sum_{p=-\infty}^{p=\infty} \frac{q^{\frac{1}{2}(2 p+1)}}{\left(1-q^{2 p+1}\right)^{2}} .
$$


Von den beiden Summen rechts in vorstehender Gleichung ist die erste offenbar $=0$, die zweite

also nach (10.), (15.) und (17.)

$$
=\sum_{p=0}^{p=\infty} \frac{q^{1}(2 p+1)\left(1+q^{2 p+1}\right)}{\left(1-q^{2 p+1}\right)^{2}},
$$

$$
=k \cdot\left(\frac{K}{\pi}\right)^{2} \text {. }
$$

Während also im allgemeinen der Factor von $-\frac{1}{2} 2^{\frac{1}{2}(2 m+1)} v^{\frac{1}{2}(2 n+1)}$ gleich

$$
-\frac{K}{\pi} \cdot \frac{q^{\frac{1}{2(2 m+1)}}}{\left(1+q^{2 m+1}\right)} \frac{q^{\frac{1}{2(2 n+1)}}}{\left(1+q^{2 n+1}\right)}
$$

ist, so wird er für $m=n$ gleich dem obigen Werthe, vermehrt um

folglich erhält man

$$
\frac{k K^{2}}{\pi^{2}} \cdot \frac{q^{\frac{1}{2(2 m+1)}}}{1+q^{2 m+1}}
$$

$$
\sin f(x) \sin f(y) \Delta f(x+y)=\cos f(x) \cos f(y)-\cos f(x+y)
$$

oder

$$
\cos f(x+y)=\cos f(x) \cos f(y)-\sin f(x) \sin f(y) \Delta f(x+y) .
$$

Auch aus dieser Formel findet man ohne Mühe die Ausdrücke für $\cos f(x+y)$, $\Delta f(x+y)$ und hieraus für $\sin f(x+y)$, in der gewöhnlichen Gestalt.

\section{Verzeichnifs der Druckfehler in meiner Abhandlung in diesem Journal} Band 34. p. 285 et seq.

S. 306 Z. 7 v. o. im Nenner 1. m. $(1-q)\left(1-q^{2}\right)\left(1-q^{\gamma} x\right)\left(1-q^{\gamma+1} x\right)$ statt

$$
(1-q)\left(1-q^{\alpha}\right)\left(1-q^{\gamma} x\right)\left(1-q^{\gamma+1} x\right)
$$

- $306-1$ v. u. sind die Zähler der drei Glieder nicht $1,1,1$, sondern 1, $q^{2}, q^{4}$.

$-308-7$ v. o. l. m. mit $\varphi\left(\alpha, 1,1, q, \frac{q^{\frac{1}{2}-\alpha}}{z}\right)$ st. mit $\varphi\left(\alpha, 1,1, q, q^{\frac{1}{2}-\alpha} z\right)$

- 313 - 6 v. o., Z. 1. v. u., S. 314 Z. 2 v. o. 1. m. auf der rechten Seite der Gleichungen respective

$$
\begin{aligned}
& h \cdot \frac{e^{\left(\frac{1}{2} \pi-b\right)(n-1) i}}{q^{\frac{1}{4}(n-1)^{2}}} \cdot H\left(q, \frac{2 K b}{\pi}\right), \\
& h \cdot \frac{e^{\left(\frac{1}{2} \pi-b\right)(n-1) i}}{q^{\frac{3}{4}(n-1)^{2}}} \cdot \Theta\left(q, \frac{2 K b}{\pi}\right), \\
& h \cdot \frac{e^{\left(\frac{1}{2} \pi-b\right)(n-1) i}}{q^{\frac{3}{4}(n-1)^{2}}} \cdot H\left(q, \frac{2 K b}{\pi}\right) .
\end{aligned}
$$

$-319-1$ v. o. statt und 1. m. also

- $324^{\circ}-4$ v. u. statt folgender Gleichungen l. m. die Gleichung (I.)

- $326-10$ v. o. statt $r^{m}, r^{2 m}$ l. m. $r, r^{2}$

- $326-12$ v. o. stalt $\cos x, \cos m x$ l. m. $r \cos 2 x, r^{m} \cos 2 m x$

- $326-10$ v. u. statt $\varphi(2 \alpha-1,1,1, q, r)$ l. m. $\varphi\left(2 \alpha-1,1,1, q, r^{2}\right)$

$-328-13$ v. 0. statt $=\varphi$ etc. 1 . m. $=\left(1-q^{1-\gamma}\right) \varphi$ etc. 